\title{
Q.
QNEEN'S
UNIVERSITY
BELFAST
}

\section{Investigation on Economic and Reliable Operation of Meshed MTDC/AC Grid as Impacted by Offshore Wind Farms}

Bu, S. Q., Du, W., Wang, H. F., Liu, Y., \& Liu, X. (2017). Investigation on Economic and Reliable Operation of Meshed MTDC/AC Grid as Impacted by Offshore Wind Farms. IEEE Transactions on Power Systems. https://doi.org/10.1109/TPWRS.2016.2636181

\section{Published in:}

IEEE Transactions on Power Systems

\section{Document Version:}

Peer reviewed version

Queen's University Belfast - Research Portal:

Link to publication record in Queen's University Belfast Research Portal

\section{Publisher rights}

Copyright 2016 IEEE. Personal use of this material is permitted. Permission from IEEE must be obtained for all other users, including reprinting/ republishing this material for advertising or promotional purposes, creating new collective works for resale or redistribution to servers or lists, or reuse of any copyrighted components of this work in other works

\section{General rights}

Copyright for the publications made accessible via the Queen's University Belfast Research Portal is retained by the author(s) and / or other copyright owners and it is a condition of accessing these publications that users recognise and abide by the legal requirements associated with these rights.

Take down policy

The Research Portal is Queen's institutional repository that provides access to Queen's research output. Every effort has been made to ensure that content in the Research Portal does not infringe any person's rights, or applicable UK laws. If you discover content in the Research Portal that you believe breaches copyright or violates any law, please contact openaccess@qub.ac.uk. 


\title{
Investigation on Economic and Reliable Operation of Meshed MTDC/AC Grid as Impacted by Offshore Wind Farms
}

\author{
S. Q. Bu, W. Du, Member, IEEE, H. F. Wang, Senior Member, IEEE, Y. Liu, and X. Liu, Member, \\ IEEE
}

\begin{abstract}
The influence of complementarity, balancing, connected grid and control of offshore wind power generation on the economic and reliable operation of hybrid multi-terminal HVDC (MTDC) and AC power system is investigated in the paper. The variances of distribution curve of both transmission loss of AC/DC system and voltage of critical nodes assessed by cumulantbased method are used to demonstrate how the different complementarity conditions, generation balancing strategies, MTDC grid topologies and control schemes affects power system operation. In this paper, a study case of 16 -generator 68 -bus power system connected with three offshore wind farms is presented. The variance indices of transmission loss and voltage magnitude are calculated and then compared under different complementarity levels, active power balancing strategies, HVDC transmission systems and controls respectively. The analysis reveals that higher complementarity and multi-machine balancing strategy can effectively reduce system operational cost and enhance operational reliability. Compared to the point-to-point topology, the control pattern and parameter setting of multi-terminal topology are two key factors to determine its impact on system operation.
\end{abstract}

Index Terms-Balancing, complementarity, cumulant, multiterminal HVDC, offshore wind power generation, system operation, variance indices.

\section{INTRODUCTION}

$\mathrm{T}_{\mathrm{s}}$ HE integration of variable wind power sources introduces stochastic power fluctuation into the electricity transmission network, which has a significant impact on the economic and reliable operation of traditional power system. The fluctuation pattern of wind generation usually varies with different regions, which can be employed to mitigate the impact of power stochastic variation brought about by wind generation on the connected systems. Therefore, the concept of "complementarity" of wind power is established and related to the wind farms in different areas of power system providing their complementary capability over certain period. The application of above-mentioned complementarity has been fully appreciated and investigated dating back to 1979 . The initial thought of combining geographically dispersed wind farms to level the total wind power output is proposed by [1], and then more research efforts have been devoted to put this

S. Q. Bu (corresponding author) is with the Department of Electrical Engineering, The Hong Kong Polytechnic University, Kowloon, H.K. (e-mail: siqi.bu@polyu.edu.hk).W.Du and H. F. Wang are with the School of Electrical and Electronic Engineering, North China Electric Power University, Beijing, idea into practice recently [2]-[6]. Based on the real historical wind data, it is demonstrated that both US [2][3] and China [4] bulk transmission systems could benefit from the interconnection of wind power from multiple sites to obtain a stable power supply from wind energy. [5] investigates the possibility to extend the application to complement different types of renewable energy (e.g., wind and solar energy), which are integrated to the different areas of power grid. In [6], the complementarity of wind power and relevant issues were intensively studied in the aspect of probabilistic small-signal stability, rather than probabilistic load flow analysis that focuses on power system operation. Hence, there is no systematic study up to date to explain the impact mechanism of complementarity of wind power on the economical efficiency and reliability of power system operation in any previous publications of probabilistic load flow analysis [7]-[9] or probabilistic optimal load flow analysis [10][11].

To connect the increasing number of offshore wind farms, VSC (Voltage Source Converter)-HVDC technology has demonstrated its superiority to the conventional AC or LCC (Line Commutated Converter)-HVDC transmission network, due to the flexibility in topology selections and control of power flow direction [12]. Compared with the mature point-to-point HVDC (PTPDC) connection for offshore wind power, multiterminal HVDC (MTDC) technology is more capable in sharing and transmitting bulk wind power from multiple areas [13][14]. Master-slave control (MSC) and DC voltage droop control (VDC) schemes are two major control patterns for VSC-HVDC. The former is derived from PTPDC network and then extended to MTDC. The latter developed specifically for MTDC owns more advantages [15]. To date, some research [16][17] has been carried out to analyze the general economical efficiency of different network topologies and control strategies of MTDC for offshore wind farms, which is however the deterministic analysis, not associated with the stochastic uncertainty of wind power sources. No study has been published yet to analyze the effect of different HVDC connections and controls on either economical efficiency or reliability of system operation when considering power variation caused by offshore wind farms.

For the simplicity of the analysis, the variation of wind power

China. Y. Liu is with the Institute of Electrical Engineering, Chinese Academy of Sciences, Beijing, China. X. Liu is with the School of Electronics, Electrical Engineering and Computer Science, Queen's University Belfast, Belfast, U.K.. 
output is usually compensated by the slack machine in the system. However, this simplification does not actually align with the real case of system operation. Especially with high penetration of wind power, it is technically difficult to achieve this simplification and multiple conventional synchronous machines are required as a reserve to participate in the system balancing depending on their availability and balancing price in the real time. Therefore, it is worthy to carefully examine the impact of different balancing strategies of offshore wind generation on system operation.

Considering the above points, complementarity, balancing, grid connection and control are examined extensively by this work, which are four major integration aspects of offshore wind generation to affect the system operation. The rest of the paper is structured as follows. Firstly, the variances of probability density function (PDF) of transmission loss and voltage magnitude of critical branches are derived respectively in Section II, which are used as two major evaluation indices of system economic and reliable operation to replace conventional PDFs for more efficient analysis. Section III presents a comprehensive study of 16-machine 68-bus power system with three offshore wind farms. Firstly, the variance indices are calculated for PTPDC under the scenario of two complementarity conditions and two balancing strategies. Then MTDC transmission is studied with different control options. Two locations of DC voltage converter are considered for MSC and two DC voltage droop constants are considered for the two types of VDC. To validate the variance assessment, Monte Carlo (MC) simulation is employed in each case.

\section{VARIANCE INDICES FOR ECONOMICAL EFFICIENCY AND RELIABILITY OF POWER SYSTEM OPERATION}

Based on statistics theory [18][19], providing the linear relation is established between one variable $\rho$ and $m$ other variables $\eta_{j}, j=1,2, \cdots m$, (i.e., $\rho=a_{1} \eta_{1}+a_{2} \eta_{2}+\cdots+a_{m} \eta_{m}$ ), their $2^{\text {nd }}$ order cumulants should satisfy

$$
\gamma_{\rho}^{(2)}=\sum_{j 1=1}^{m} \sum_{j 2=1}^{m} a_{j_{1}} a_{j_{2}} \gamma_{\eta_{j 1 j 2}}^{(2)}
$$

where $\gamma_{\rho}^{(2)}$ represents the $2^{\text {nd }}$ order cumulant of $\rho$ and $\gamma_{\eta_{j_{1} / 2}}^{(2)}$ represents the $2^{\text {nd }}$ order cross cumulant between $\eta_{j}, j=1,2, \cdots m$.

As the variance of $\rho, \sigma_{\rho}^{2}$, is equal to $\gamma_{\rho}^{(2)}$ and the covariance of $\eta_{j 1}$ and $\eta_{j 2}, \operatorname{cov}\left(\eta_{j 1}, \eta_{j 2}\right)$, is equal to $\gamma_{\eta_{j_{1} / 2}}^{(2)}$, the relationship between the variance of $\rho$ and the covariance of $\eta_{j 1}$ and $\eta_{j 2}$ can be established by (1).

The cumulant-based method above is used to evaluate the variance indices of system operation and the variance index of composite wind power ( $\left.\sigma_{P_{w c}}^{2}\right)$ proposed in [6] is also used as an indicator of complementarity level in this paper.

\section{A. Variance index for power system transmission loss}

The transmission loss of power system is a critical index to measure the economical efficiency of system operation. Power system planner and operator set up the annual target and try to keep the power loss within certain percentage of total demand [20]. The probability that the transmission loss can stay within certain standard can be computed by using the PDF of transmission loss, which closely relies on the mean and variance of PDF of transmission loss. The mean of transmission loss mainly determined by the average wind speed and the variance of probabilistic distribution of transmission loss can be assessed as follows.

The transmission loss $P_{\text {loss }}$ is equal to the total sum of active power injection at all nodes as shown below

$$
P_{\text {loss }}=\sum_{i=1}^{n} P_{i}
$$

where $P_{i}$ is the active power injection of the $i^{t h}$ node (either $\mathrm{AC}$ or DC node) and $n$ is the total number of hybrid AC/DC system node. Hence, $P_{\text {loss }}$ is also equal to the sum of $\mathrm{AC}$ network loss and DC network loss (including loss from VSC), i.e., $P_{\text {loss }}=P_{\text {ACloss }}+P_{\text {DCloss }} . P_{i}$ is positive if $i^{t h}$ node is a generation node and negative if $i^{t h}$ node is a load node.

Assuming $m$ offshore wind farms connected to the system, the relationship between the transmission loss and each offshore wind power is established

$$
\begin{aligned}
\Delta P_{\text {loss }} & =\sum_{j=1}^{m}\left[\left(\partial P_{\text {loss }} / \partial P_{w j}\right) \Delta P_{w j}\right] \\
& =\sum_{j=1}^{m}\left[\left(\frac{\partial\left(\cdots+P_{\text {slack }}+\cdots+P_{w j}+\cdots\right)}{\partial P_{w j}}\right) \Delta P_{w j}\right] \\
& =\sum_{j=1}^{m}\left[\left(\partial P_{\text {slack }} / \partial P_{w j}+1\right) \Delta P_{w j}\right]
\end{aligned}
$$

where $P_{\text {slack }}$ denotes the power injection from the slack machine, and $P_{w j}$ represents the $j^{\text {th }}$ wind power, $j=1,2, \cdots m$. The derivation of (3) is based on the assumption that the variation of wind power $P_{w j}$ is only compensated by the slack machine and the power output of other wind power, synchronous machines and loads are unchanged and independent from $P_{w j}$.

During high level penetration of wind generation, each wind power variation might be compensated by different combinations of conventional synchronous machines. The multi-machine balancing strategy can be expressed as

$$
\begin{gathered}
\Delta P_{w j}+\Delta P_{g 1}+\Delta P_{g 2}=0 \\
\Delta P_{w j}=-\Delta P_{g 1} / \alpha_{1} \\
\Delta P_{w j}=-\Delta P_{g 2} / \alpha_{2}
\end{gathered}
$$

where the $j^{\text {th }}$ wind power is compensated by two machines $g 1$ and $g 2$ as an example with the compensation coefficient $\alpha_{1}$ and $\alpha_{2}$ respectively. The sum of all the compensation coefficients should be equal to one. Thus, when multi-machine balancing strategy in (4) is adopted, $\Delta P_{\text {loss }}$ becomes

$$
\begin{aligned}
\Delta P_{\text {loss }} & =\sum_{j=1}^{m}\left[\left(\partial P_{\text {loss }} / \partial P_{w j}\right) \Delta P_{w j}\right] \\
& =\sum_{j=1}^{m}\left[\left(\frac{\partial\left(\cdots P_{g 1}+P_{g 2}+\cdots+P_{\text {slack }}+\cdots+P_{w j}+\cdots\right)}{\partial P_{w j}}\right) \Delta P_{w j}\right] \\
& =\sum_{j=1}^{m}\left[\left(\partial P_{\text {slack }} / \partial P_{w j}+1-\alpha_{1}-\alpha_{2}\right) \Delta P_{w j}\right] \\
& =\sum_{j=1}^{m}\left[\left(\partial P_{\text {slack }} / \partial P_{w j}\right) \Delta P_{w j}\right]
\end{aligned}
$$

$\partial P_{\text {slack }} / \partial P_{w j}$ in (3) and (5) could be calculated numerically by simply applying a $1 \%$ of perturbation on the $j^{\text {th }}$ wind active power. On this basis, the sensitivity of power loss with respect 
to the $j^{\text {th }}$ wind power (i.e., $\partial P_{\text {loss }} / \partial P_{w j}$ ) under different balancing strategies can be determined by (3) and (5) respectively.

By applying (1), PDF variance of $\Delta P_{\text {loss }}$ can be written as

$$
\begin{aligned}
\sigma_{\Delta P_{\text {loss }}}^{2} & =\gamma_{\Delta P_{\text {loss }}}^{(2)} \\
& =\sum_{j 1=1}^{m} \sum_{j 2=1}^{m}\left(\partial P_{\text {loss }} / \partial P_{w j 1}\right)\left(\partial P_{\text {loss }} / \partial P_{w j 2}\right) \gamma_{\Delta P_{w j 1 j 2}}^{(2)}
\end{aligned}
$$

Since $\sigma_{P_{l o s s}}^{2}=\sigma_{\Delta P_{l o s s}}^{2}$ and $\sigma_{P_{w j}}=\sigma_{\Delta P_{w j}}$,

$$
\begin{aligned}
\sigma_{P_{\text {loss }}}^{2}= & {\left[\left(\frac{\partial P_{\text {loss }}}{\partial P_{w 1}}\right) \sigma_{P_{w 1}}\right]^{2}+\cdots+\left[\left(\frac{\partial P_{\text {loss }}}{\partial P_{w m}}\right) \sigma_{P_{w m}}\right]^{2} } \\
& +2\left(\frac{\partial P_{\text {loss }}}{\partial P_{w 1}}\right)\left(\frac{\partial P_{\text {loss }}}{\partial P_{w 2}}\right) \rho_{P_{w 1} P_{w 2}} \sigma_{P_{w 1}} \sigma_{P_{w 2}}+\cdots \\
& +2\left(\frac{\partial P_{\text {loss }}}{\partial P_{w m-1}}\right)\left(\frac{\partial P_{\text {loss }}}{\partial P_{w m}}\right) \rho_{P_{w m-1} P_{w m}} \sigma_{P_{w m-1}} \sigma_{P_{w m}}
\end{aligned}
$$

where the correlation coefficient (CC) $\rho_{P_{w j 1} P_{w j 2}}$ and the standard deviation (SD) $\sigma_{P_{w j}}$ are determined by wind speed distribution. Usually, the wind power from different regions should have a positive correlation $\left(\rho_{P_{w j 1} P_{w j}} \in[0,1]\right)$. As stated in (3) and (5), $\partial P_{l o s s} / \partial P_{w j}$ varies with different balancing strategies.

Therefore, the PDF variance of transmission loss, $\sigma_{P_{\text {loss }}}^{2}$, is assessed by (7) as an index of economic operation. It can be seen that $\sigma_{P_{\text {loss }}}^{2}$ relies on the sensitivities of $P_{\text {loss }}$ in respect of each wind active power, the CCs among different wind power as well as the SD of $P_{w j}$.

If the transmission loss of the system under the average wind speed condition is within the annual target threshold, the smaller $\sigma_{P_{\text {loss }}}^{2}$ produces a higher probability of economic operation as the shape of PDF curve is largely affected by the variance. As $\mathrm{SD}$ and $\mathrm{CC}$ are related to the complementarity level of wind power [6], and sensitivity of transmission loss is influenced by balancing strategy, HVDC transmission structure and converter control scheme, $\sigma_{P_{\text {loss }}}^{2}$ derives a relation between these four aspects of offshore wind generation and economical efficiency of power systems.

\section{B. Variance index for voltage magnitude of critical nodes}

According to the National Electricity Transmission System Security and Quality of Supply Standards (SQSS) [21], there should not be any steady-state voltage violation at system nodes of all levels or any voltage step change violation at Grid Supply Points (GSPs). Therefore, system voltage, as a major indicator of supply reliability, needs to be maintained within operational limits. Due to a lack of support in the reactive compensation, voltage at some critical nodes may easily breach the required standard when wind power of high penetration level fluctuates as revealed in previous research [7]-[9]. To keep the system voltage between upper and lower operational limits, the variance of probabilistic distribution of the voltage needs to be below certain level since the variance could affect the probability of voltage violation.

The relation between the voltage magnitude of critical nodes and offshore wind power can be computed to be

$$
\Delta V_{c}=\sum_{j=1}^{m}\left[\left(\partial V_{c} / \partial P_{w j}\right) \Delta P_{w j}\right]
$$

where $V_{c}$ denotes the voltage magnitude at critical nodes, and $\partial V_{c} / \partial P_{w j}$ represents the sensitivity of $V_{c}$ regarding $P_{w j}$. The sensitivity of $V_{c}$ in respect of the $m$ wind power at the equilibrium point in (8) could be calculated either in the analytical way by using the inverse Jacobian matrix [8] or in the numerical way. Based on the derivation in [8], it can be easily proved that the balancing strategy of wind power variation will affect the sensitivity results.

Similar to (7), the PDF variance of $V_{c}$ can be derived as

$$
\begin{aligned}
\sigma_{V_{c}}^{2}= & {\left[\left(\frac{\partial V_{c}}{\partial P_{w 1}}\right) \sigma_{P_{w 1}}\right]^{2}+\cdots+\left[\left(\frac{\partial V_{c}}{\partial P_{w m}}\right) \sigma_{P_{w m}}\right]^{2} } \\
& +2\left(\frac{\partial V_{c}}{\partial P_{w 1}}\right)\left(\frac{\partial V_{c}}{\partial P_{w 2}}\right) \rho_{P_{w 1} P_{w 2}} \sigma_{P_{w 1}} \sigma_{P_{w 2}}+\cdots \\
& +2\left(\frac{\partial V_{c}}{\partial P_{w m-1}}\right)\left(\frac{\partial V_{c}}{\partial P_{w m}}\right) \rho_{P_{w m-1} P_{w m}} \sigma_{P_{w m-1}} \sigma_{P_{w m}}
\end{aligned}
$$

It is revealed by (9) that similar to $\sigma_{P_{\text {loss }}}^{2}$, the PDF variance of $V_{c}, \sigma_{V_{c}}^{2}$, also depends on the sensitivities of $V_{\mathrm{c}}$, the CCs among different wind power and the SD of $P_{w j}$. Therefore, (9) can be used to demonstrate how these four aspects mentioned previously affect the probability of voltage violation and thus operational reliability.

\section{VSC-MTDC MODELING AND CONTROL [13][15]}

\section{A. Converter Model}

Although the modular multilevel converter (MMC) approach based VSC-HVDC does not require the filtering function, without loss of generality, the VSC-MTDC converter in this paper is installed with transformer, AC filter and phase reactor. In steady state, the converter is modeled as a controllable voltage source $U_{c k} \angle \theta_{c k}$ behind transformer impedance $\bar{Z}_{t k}$, phase reactor impedance $\bar{Z}_{c k}$ and AC filter susceptance $B_{f k}$ shown in Fig. 1. Therefore, the apparent power injection into the AC grid from the $k^{\text {th }}$ converter is denoted as $\bar{S}_{s k}=P_{s k}+$ $j Q_{s k}$ and can be computed

$P_{s k}=-U_{s k}^{2} G_{t k}+U_{s k} U_{f k}\left[G_{t k} \cos \left(\delta_{s k}-\delta_{f k}\right)+B_{t k} \sin \left(\delta_{s k}-\delta_{f k}\right)\right]$

$Q_{s k}=U_{s k}^{2} B_{t k}+U_{s k} U_{f k}\left[G_{t k} \sin \left(\delta_{s k}-\delta_{f k}\right)-B_{t k} \cos \left(\delta_{s k}-\delta_{f k}\right)\right]$

where $G_{t k}+j B_{t k}=1 / \bar{Z}_{t k}$ is the admittance of the transformer. The power flow at the AC filter and converter buses can be also computed

$$
\begin{aligned}
& P_{c k}=-U_{c k}^{2} G_{c k}-U_{f k} U_{c k}\left[G_{c k} \cos \left(\delta_{f k}-\delta_{c k}\right)-B_{c k} \sin \left(\delta_{f k}-\delta_{c k}\right)\right] \\
& Q_{c k}=-U_{c k}^{2} B_{c k}+U_{f k} U_{c k}\left[G_{c k} \sin \left(\delta_{f k}-\delta_{c k}\right)+B_{c k} \cos \left(\delta_{f k}-\delta_{c k}\right)\right] \\
& P_{f s k}=U_{f k}^{2} G_{t k}-U_{f k} U_{s k}\left[G_{t k} \cos \left(\delta_{s k}-\delta_{f k}\right)-B_{t k} \sin \left(\delta_{s k}-\delta_{f k}\right)\right] \\
& Q_{f s k}=-U_{f k}^{2} B_{t k}+U_{f k} U_{s k}\left[G_{t k} \sin \left(\delta_{s k}-\delta_{f k}\right)+B_{t k} \cos \left(\delta_{s k}-\delta_{f k}\right)\right] \\
& P_{c f k}=-U_{f k}^{2} G_{c k}+U_{f k} U_{c k}\left[G_{c k} \cos \left(\delta_{f k}-\delta_{c k}\right)+B_{c k} \sin \left(\delta_{f k}-\delta_{c k}\right)\right] \\
& Q_{c f k}=U_{f k}^{2} B_{c k}+U_{f k} U_{c k}\left[G_{c k} \sin \left(\delta_{f k}-\delta_{c k}\right)-B_{c k} \cos \left(\delta_{f k}-\delta_{c k}\right)\right]
\end{aligned}
$$

where $G_{c k}+j B_{c k}=1 / \bar{Z}_{c k}$ is the admittance of the phase reactor. The reactive power of $A C$ filter can be computed to be 


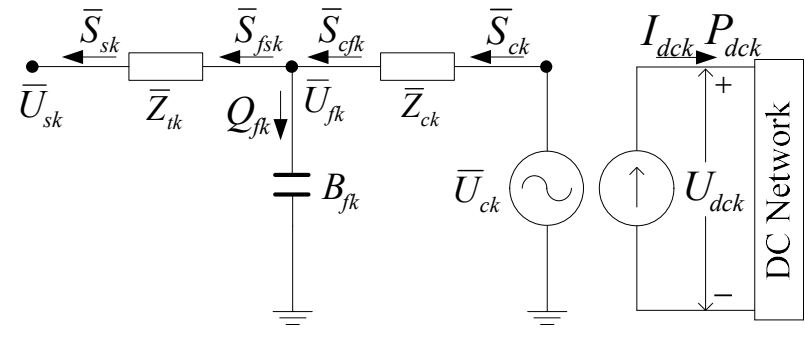

Fig. 1. Equivalent model of the $k^{\text {th }}$ converter connecting the $\mathrm{AC}$ and $\mathrm{DC}$ grid.

$$
Q_{f k}=-U_{f k}^{2} B_{f k}
$$

If the MMC approach is employed, the AC filter is removed and hence the transformer and phase reactor can be lumped together which will simplify the equations above.

For the $k^{\text {th }}$ converter, it should have

$$
P_{c k}+P_{d c k}+P_{\text {lossk }}=0
$$

where $P_{c k}$ and $P_{d c k}$ is the active power injected by the $k^{\text {th }}$ converter into the AC and DC grid respectively, and $P_{\text {lossk }}$ is the converter losses, which is related to the AC current of the $k^{\text {th }}$ converter.

$$
P_{\text {lossk }}=a_{k}+b_{k} I_{c k}+c_{k} I_{c k}^{2}
$$

where $I_{c k}=\sqrt{P_{c k}^{2}+Q_{c k}^{2}} / U_{c k}$, and $a_{k}, b_{k}$ and $c_{k}$ are the loss parameters.

\section{B. DC Grid Model}

The DC grid model consists of DC current and power equations. The DC current equation can be written as

$$
I_{d c l}=\sum_{\substack{m=1 \\ m \neq l}}^{n_{d c}} Y_{d c l m}\left(U_{d c l}-U_{d c m}\right) \quad \forall l \in N_{d c}
$$

where $Y_{d c l m}$ is the element of admittance matrix of DC grid, $n_{d c}$ is the total bus number of DC grid, $N_{d c}$ is the DC bus set, $I_{d c l}$ is the current injection into the $l^{t h} \mathrm{DC}$ bus and $U_{d c l}$ is the terminal voltage of the $l^{\text {th }} \mathrm{DC}$ bus. Hence, DC power equation for a bipole DC grid can be obtained

$$
P_{d c l}=2 U_{d c l} I_{d c l} \quad \forall l \in N_{d c}
$$

\section{VSC-MTDC Control Schemes}

As reviewed in Section I, the two major control strategies, i.e., master-slave control (MSC) and DC voltage droop control (VDC), are employed in this work. Compared with MSC, VSC is more reliable and flexible in facilitating the converter outage of DC grid.

For MSC strategy, the converter can select either Constant $P_{S}$ mode (for DC non-slack bus) or Constant $U_{d c}$ mode (for DC slack bus) and normally there is only one slack bus in one DC grid. To control the reactive power on the AC side of VSC in an independent manner, the converter should also select either Constant $Q_{s}$ or $U_{s}$ mode. Hence, there are totally four types of mode combination (i.e., $P_{s} Q_{s}, P_{s} U_{s}, U_{d c} Q_{s}$ and $U_{d c} U_{s}$ ) for each converter.

For VSC strategy, there are two typical schemes, namely injected DC power based voltage droop control (PVDC) and injected DC current based voltage droop control (CVDC), as illustrated by Fig. 2(a) and 2(b) separately. In Fig. 2, PVDC and CVDC operate in the rectifier and inverter mode respectively as an example. $k_{\text {droop }}$ is the droop constant, and the suffix $r e f$ denotes the reference value of relevant variables.

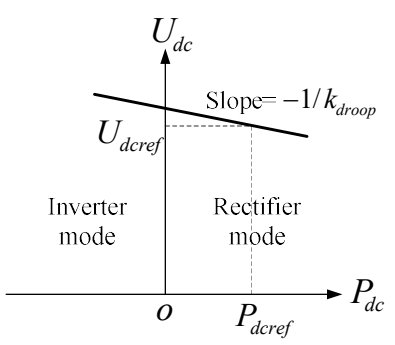

(a) PVDC

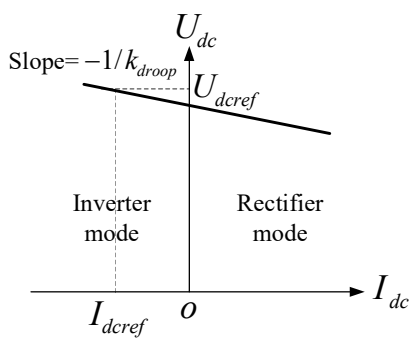

(b) CVDC
Fig. 2. DC voltage droop control schemes of VSC.

\section{CASE STUdy}

The power system operation analysis proposed above is tested in a 16-generator 68-bus power system [22] connected with three offshore installed wind farms displayed in Fig. 3. As the connected location is not discussion focus of the paper, bus 23, 24 and 29 are chosen as the three wind power injection buses. Two typical topologies of VSC-HVDC grid to integrate three wind farms shown in Fig. 4 are investigated. The parameters of AC network and synchronous machine are available in [22]. The detailed model of wind power generator in [23] is employed. The parameters of the wind power generators, wind speed distributions and power-wind speed curve data are presented in Appendix A. The detailed model of VSC and its control strategies in Section III are used and parameters of VSC-HVDC network and converter are provided in Appendix B.

Seven sequential cases are presented below for a comprehensive comparison study. To facilitate the understanding of each comparison, the full settings of seven study cases are given in Table I.

TABLE I

SetTing Summary of EACH Study CASE

\begin{tabular}{|l|c|c|c|c|c|c|c|}
\hline & $\begin{array}{c}\text { Case } \\
\text { A }\end{array}$ & $\begin{array}{c}\text { Case } \\
\text { B }\end{array}$ & $\begin{array}{c}\text { Case } \\
\text { C }\end{array}$ & $\begin{array}{c}\text { Case } \\
\text { D }\end{array}$ & $\begin{array}{c}\text { Case } \\
\text { E }\end{array}$ & $\begin{array}{c}\text { Case } \\
\text { F }\end{array}$ & $\begin{array}{c}\text { Case } \\
\text { G }\end{array}$ \\
\hline LC & $\sqrt{ }$ & & & & & & \\
\hline HC & & $\sqrt{ }$ & $\sqrt{ }$ & $\sqrt{ }$ & $\sqrt{ }$ & $\sqrt{ }$ & $\sqrt{ }$ \\
\hline PTPDC & $\sqrt{ }$ & $\sqrt{ }$ & $\sqrt{ }$ & & & & \\
\hline MTDC & & & & $\sqrt{ }$ & $\sqrt{ }$ & $\sqrt{ }$ & $\sqrt{ }$ \\
\hline SB & $\sqrt{ }$ & $\sqrt{ }$ & & & & & \\
\hline MB & & & $\sqrt{ }$ & $\sqrt{ }$ & $\sqrt{ }$ & $\sqrt{ }$ & $\sqrt{ }$ \\
\hline MSC & & & & $\sqrt{ }$ & $\sqrt{ }$ & & \\
\hline DC5 & & & & $\sqrt{ }$ & & & \\
\hline DC6 & & & & & $\sqrt{ }$ & & \\
\hline 2VDCs & & & & & & $\sqrt{ }$ & $\sqrt{ }$ \\
\hline HDCC & & & & & & $\sqrt{ }$ & \\
\hline LDCC & & & & & & & $\sqrt{ }$ \\
\hline
\end{tabular}

Table Note:

LC $=$ Low Complementarity Level

$\mathrm{HC}=$ High Complementarity Level

$\mathrm{SB}=$ Slack Machine Balancing

$\mathrm{MB}=$ Multiple Machine Balancing

DC5 $=$ DC5 as DC Slack Bus for MSC

DC6 $=$ DC6 as DC Slack Bus for MSC

2VDCs = Two Types of VDC Strategies

HDCC $=$ High Droop Control Constant

LDCC $=$ Low Droop Control Constant 


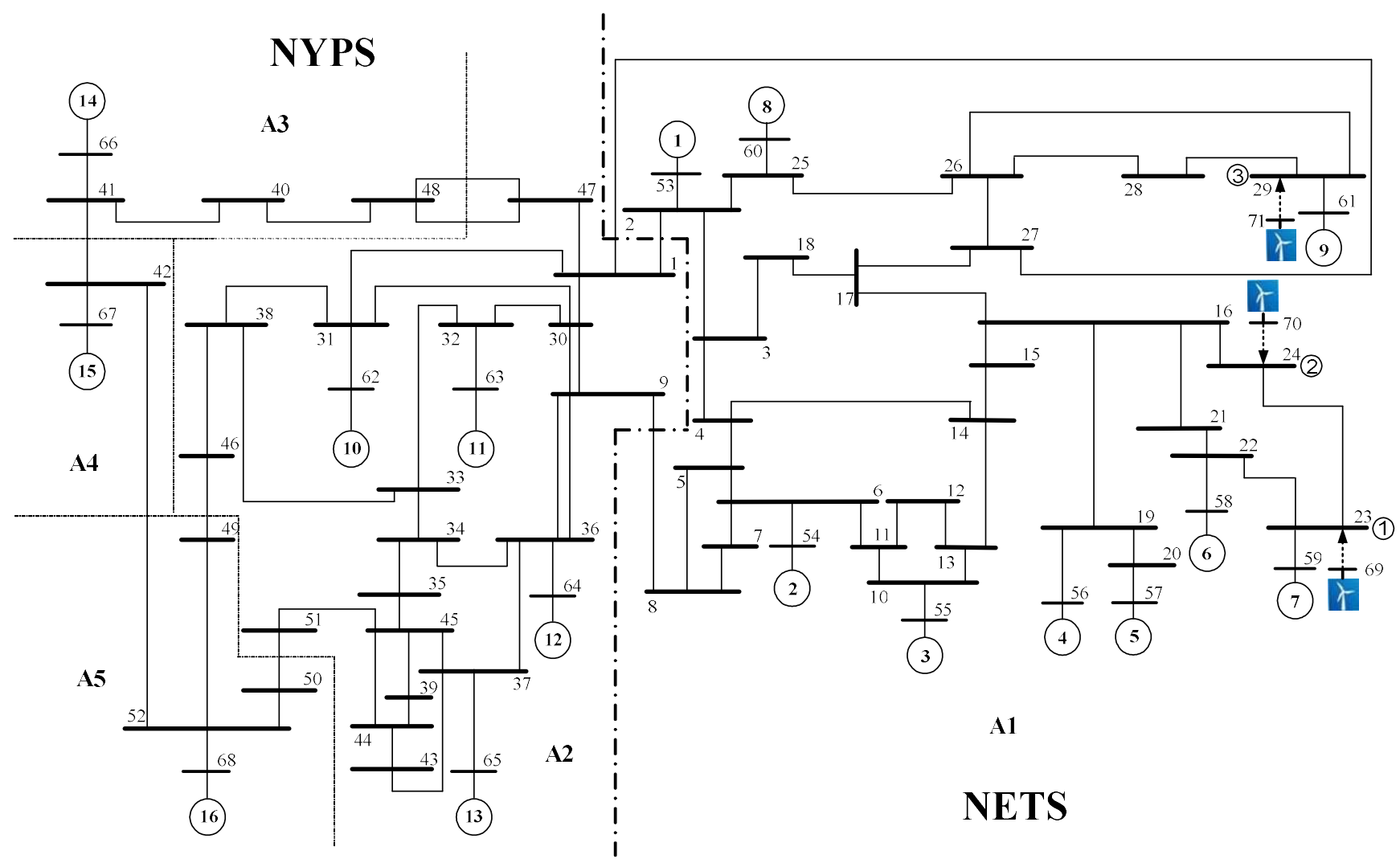

Fig. 3. 16-generator 68 -bus test power system integrated with 3 offshore wind farms.

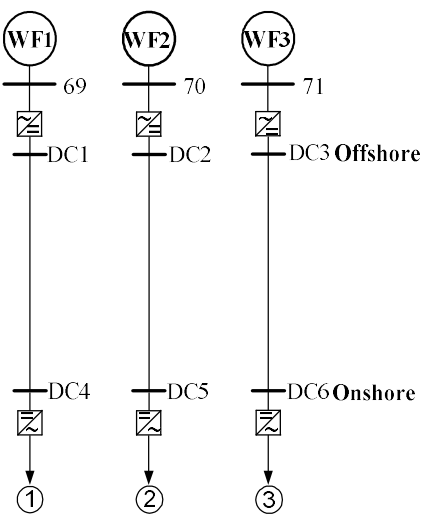

(a)

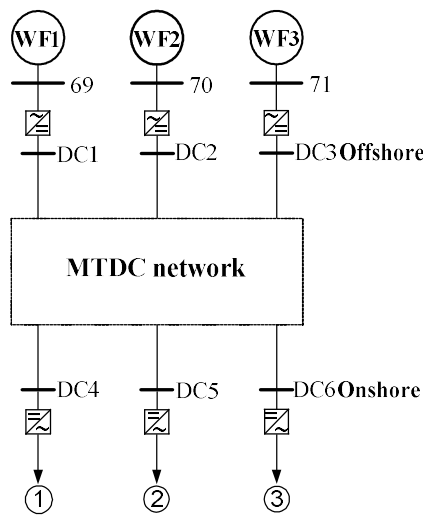

(b)

Fig. 4. Two typical VSC-HVDC transmission networks:

(a) point-to-point HVDC;

(b) multi-terminal HVDC.

\section{1) Case A (Utilization of PTPDC and slack machine} balancing):

Three PTPDC lines displayed in Fig. 4(a) connects three offshore wind farms respectively in Case A. By deterministic load flow analysis of base case, the transmission loss of test system $P_{\text {loss }}$ is 3.5620 . The transmission loss percentage $P_{\text {loss }} \%$ is defined to be $P_{\text {loss }} / P_{\text {demandtot }}$ and the annual target $P_{\text {loss }} \%$ is set at $2 \%$. As the total active power demand is assumed to be constant (i.e., $P_{\text {demandtot }}=182.3390$ ), the transmission loss allowed is 3.6468. Hence deterministically the system loss is within the target threshold. In the study, the

$8^{\text {th }}$ bus node is identified to be one of the critical nodes for monitoring the voltage. The node is a GSP and located in the middle of two subsystems (i.e., NETS and NYPS). Since there is no generator or any reactive power compensation around, the node is quite vulnerable in maintaining its voltage during significant transfer variation between two subsystems. For the base case, the voltage of the $8^{\text {th }}$ node is 0.9817 and the upper and lower voltage limit is set to $\pm 2 \%$.

The wind farms installed at bus 69, 70 and 71 are denoted as the 1st, 2nd and 3rd wind power source respectively. The variation of the wind power is balanced by slack machine only in Case A. The sensitivities of transmission loss and critical node voltage in respect of three wind generation sources are $\partial P_{\text {loss }} / \partial P_{w 1}=0.0792, \partial P_{\text {loss }} / \partial P_{w 2}=0.0640, \partial P_{\text {loss }} / \partial P_{w 3}=0.0956$ $\partial V_{c} / \partial P_{w 1}=-0.0013, \partial V_{c} / \partial P_{w 2}=-0.0012, \partial V_{c} / \partial P_{w 3}=-0.0014$ Three sample series of wind speed that follow the Weibull distribution as well as spatial correlations presented in Appendix A.2 are generated by the method proposed in [24].

Then relevant sample series of wind power can be computed by applying the power-wind speed relation curve, which are verified by the historical metering data from utilities. Thus, the $\mathrm{SD}$ and CCs of three wind power are computed

$\sigma_{P_{w 1}}=\sigma_{P_{w 2}}=\sigma_{P_{w 3}}=0.3271$,

$\rho_{P_{w 1} P_{w 2}}=0.8827, \rho_{P_{w 1} P_{w 3}}=0.2750, \rho_{P_{w 2} P_{w 3}}=0.4643$

According to [6] as well as (7) and (9), the variance indices of complementarity level, $P_{\text {loss }}$ and $V_{c}$ are computed to be $\sigma_{P_{w c}}^{2}=\sigma_{P_{w 1}}^{2}+\sigma_{P_{w 2}}^{2}+\sigma_{P_{w 3}}^{2}+2 \rho_{P_{w 1} P_{w 2}} \sigma_{P_{w 1}} \sigma_{P_{w 2}}$ 


$$
\begin{aligned}
+ & 2 \rho_{P_{w 1} P_{w 3}} \sigma_{P_{w 1}} \sigma_{P_{w 3}}+2 \rho_{P_{w 2} P_{w 3}} \sigma_{P_{w 2}} \sigma_{P_{w 3}} \\
= & 6.6825 \times 10^{-1} \\
\sigma_{P_{\text {loss }}}^{2}= & {\left[\left(\frac{\partial P_{\text {loss }}}{\partial P_{w 1}}\right) \sigma_{P_{w 1}}\right]^{2}+\left[\left(\frac{\partial P_{\text {loss }}}{\partial P_{w 2}}\right) \sigma_{P_{w 2}}\right]^{2}+\left[\left(\frac{\partial P_{\text {loss }}}{\partial P_{w 3}}\right) \sigma_{P_{w 3}}\right]^{2} } \\
& +2\left(\frac{\partial P_{l o s s}}{\partial P_{w 1}}\right)\left(\frac{\partial P_{l o s s}}{\partial P_{w 2}}\right) \rho_{P_{w 1} P_{w 2}} \sigma_{P_{w 1}} \sigma_{P_{w 2}} \\
& +2\left(\frac{\partial P_{l o s s}}{\partial P_{w 1}}\right)\left(\frac{\partial P_{l o s s}}{\partial P_{w 3}}\right) \rho_{P_{w 1} P_{w 3}} \sigma_{P_{w 1}} \sigma_{P_{w 3}} \\
& +2\left(\frac{\partial P_{l o s s}}{\partial P_{w 2}}\right)\left(\frac{\partial P_{l o s s}}{\partial P_{w 3}}\right) \rho_{P_{w 2} P_{w 3}} \sigma_{P_{w 2}} \sigma_{P_{w 3}} \\
= & 4.1018 \times 10^{-3} \\
\sigma_{V_{c}}^{2}=[ & \left.\left(\frac{\partial V_{c}}{\partial P_{w 1}}\right) \sigma_{P_{w 1}}\right]^{2}+\left[\left(\frac{\partial V_{c}}{\partial P_{w 2}}\right) \sigma_{P_{w 2}}\right]^{2}+\left[\left(\frac{\partial V_{c}}{\partial P_{w 3}}\right) \sigma_{P_{w 3}}\right]^{2} \\
+ & 2\left(\frac{\partial V_{c}}{\partial P_{w 1}}\right)\left(\frac{\partial V_{c}}{\partial P_{w 2}}\right) \rho_{P_{w 1} P_{w 2}} \sigma_{P_{w 1}} \sigma_{P_{w 2}} \\
+ & 2\left(\frac{\partial V_{c}}{\partial P_{w 1}}\right)\left(\frac{\partial V_{c}}{\partial P_{w 3}}\right) \rho_{P_{w 1} P_{w 3}} \sigma_{P_{w 1}} \sigma_{P_{w 3}} \\
+ & 2\left(\frac{\partial V_{c}}{\partial P_{w 2}}\right)\left(\frac{\partial V_{c}}{\partial P_{w 3}}\right) \rho_{P_{w 2} P_{w 3}} \sigma_{P_{w 2}} \sigma_{P_{w 3}} \\
= & 1.1569 \times 10^{-6}
\end{aligned}
$$

MC simulation (5000 iterations) is implemented and hence the estimated PDF of $P_{\text {loss }}$ and $V_{c}$ from MC simulation is plotted in Fig. 5 and 6 respectively.

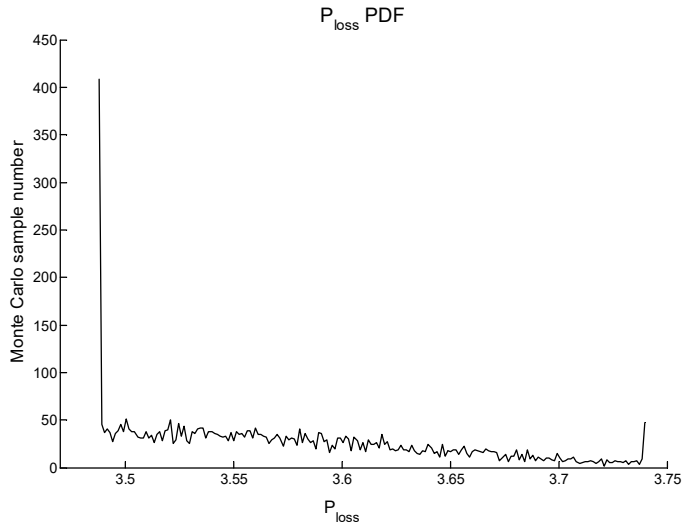

Fig. 5. Estimated PDF of $P_{\text {loss }}$ generated from MC simulation (Case A).

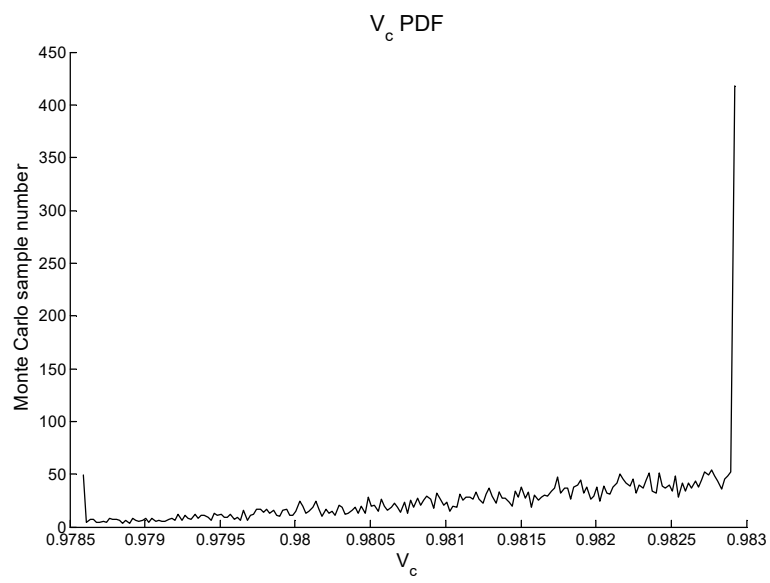

Fig. 6. Estimated PDF of $V_{c}$ generated from MC simulation (Case A).

The computing time of $\mathrm{MC}$ simulation and variance indices computation is compared. With the same computational resource (Lenovo ThinkCentre, Intel Core i7-4790 CPUs 3.60 $\mathrm{GHz}, 32.0 \mathrm{~GB}$ RAM), to compute the variance of sample data of $P_{l o s s}$ and $V_{c}$, a typical MC simulation with 5000 iterations consumes $21847.01 \mathrm{~s}$, while only $9.59 \mathrm{~s}$ for the indices computation.

Based on the computed sample data of $P_{\text {loss }}$ and $V_{c}$ from $\mathrm{MC}$ simulation, it can be calculated that

$$
\begin{aligned}
& \sigma_{P_{\text {loss }}}^{2}=4.1042 \times 10^{-3} \\
& P\left(P_{\text {loss }}<3.6468\right)=0.8366 \\
& \sigma_{V_{c}}^{2}=1.2069 \times 10^{-6} \\
& P\left(0.98<V_{c}<1.02\right)=0.8756
\end{aligned}
$$

Equation (24a) and (24c) has verified the variances assessed in (23b) and (23c). It is indicated by (24b) and (24d) that although deterministically the system is operated within the standard of economical efficiency and reliability, it has a high probability of $16.34 \%$ and $12.44 \%$ to breach the target limits caused by the low level of complementarity from composite wind power. It is also revealed by (24d) that the fluctuation of wind power could worsen the voltage of system nodes which are located even long distance away from these wind farms.

\section{2) Case B (High complementarity of wind generation applied to Case $A$ ):}

Case B selects the same load condition as Case A and hence the results of deterministic load flow analysis and sensitivity calculation do not change. By using the different wind speed distribution provided by Appendix A.2, the SD and CCs of three wind power are obtained

$$
\begin{aligned}
& \sigma_{P_{w 1}}=\sigma_{P_{w 2}}=\sigma_{P_{w 3}}=0.2860, \\
& \rho_{P_{w 1} P_{w 2}}=0.4847, \rho_{P_{w 1} P_{w 3}}=0.0003, \rho_{P_{w 2} P_{w 3}}=0.0002
\end{aligned}
$$

Then the variance indices of complementarity level, $P_{\text {loss }}$ and $V_{c}$ are computed to be

$$
\begin{aligned}
& \sigma_{P_{w c}}^{2}=3.2547 \times 10^{-1} \\
& \sigma_{P_{l o s s}}^{2}=1.9979 \times 10^{-3} \\
& \sigma_{V_{c}}^{2}=5.6241 \times 10^{-7}
\end{aligned}
$$

Compared with (23), variances in (25) are smaller due to the reduced variation and relatively weak correlations of three wind speed series. Hence the complementarity in Case B is considered as high level.

MC simulation is implemented to testify the variances assessed above and the estimated PDF of $P_{\text {loss }}$ and $V_{c}$ from MC simulation is shown in Fig. 7 and 8.

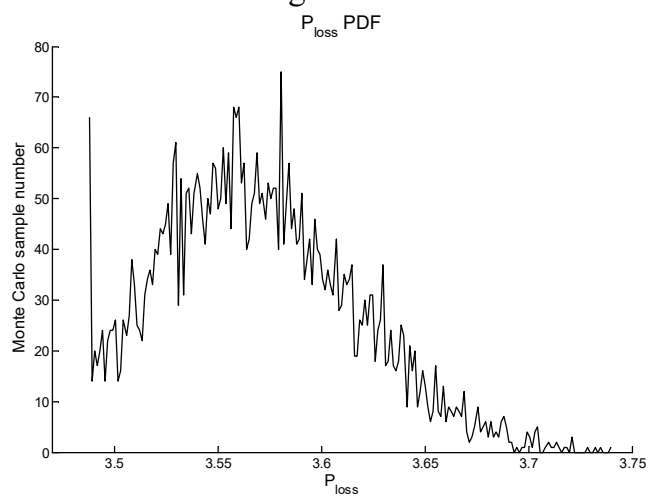

Fig. 7. Estimated PDF of $P_{\text {loss }}$ generated from MC simulation (Case B). 


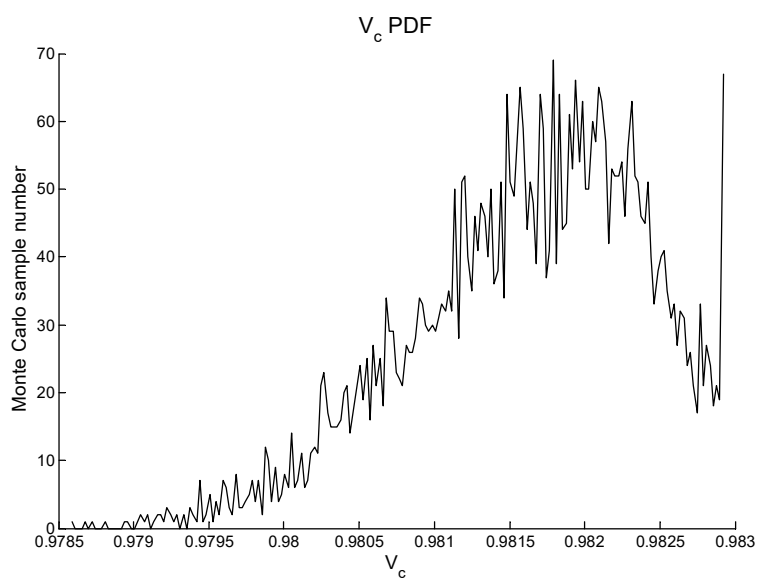

Fig. 8. Estimated PDF of $V_{c}$ generated from MC simulation (Case B).

According to the computed sample data of $P_{\text {loss }}$ and $V_{c}$ from MC simulation, it can be calculated that

$$
\begin{aligned}
& \sigma_{P_{\text {loss }}}^{2}=2.0888 \times 10^{-3} \\
& P\left(P_{\text {loss }}<3.6468\right)=0.9418 \\
& \sigma_{V_{c}}^{2}=5.9608 \times 10^{-7} \\
& P\left(0.98<V_{c}<1.02\right)=0.9672
\end{aligned}
$$

The variances in (25) have been testified by (26). Equation (26) suggests that in the high complementarity condition, the probability of transmission loss and voltage that satisfy the target criteria increases to $94.18 \%$ and $96.72 \%$ respectively due to the variance reduction of composite wind power. As a result, the effectiveness of complementarity level of wind power generation to improve system economic and reliable operation is evidently demonstrated by Case B in spite of the separate connection of offshore wind farms to the system by PTPDC transmission. That is to say, the utilization of complementarity does not need the physical combination of wind power, which is normally achieved by using MTDC network.

\section{3) Case C (Multiple machine balancing applied to Case B):}

Case $\mathrm{C}$ is set up to compare with Case B when different balancing strategy of offshore wind generation is adopted and other preconditions are not changed. The synchronous generator 11 and 12 are used to balance the wind power fluctuation with the compensation coefficients 0.43 and 0.57 respectively according to their installed capacities. The same combination of balancing machines is used in Case D-G for comparison purpose.

In this case, the results from deterministic load flow analysis as well as the level of wind power complementarity is the same as in Case B. However, due to different balancing strategies applied, as explained in Section II the sensitivity computation of transmission loss and critical node voltage in respect of three wind power sources becomes

$\partial P_{\text {loss }} / \partial P_{w 1}=0.0369, \partial P_{\text {loss }} / \partial P_{w 2}=0.0217, \partial P_{\text {loss }} / \partial P_{w 3}=0.0533$ $\partial V_{c} / \partial P_{w 1}=-0.0010, \partial V_{c} / \partial P_{w 2}=-0.0009, \partial V_{c} / \partial P_{w 3}=-0.0011$

Hence the variance indices are computed to be

$$
\begin{aligned}
& \sigma_{P_{\text {loss }}}^{2}=4.4590 \times 10^{-4} \\
& \sigma_{V_{c}}^{2}=3.1195 \times 10^{-7}
\end{aligned}
$$

Due to the decrease of absolute value of the sensitivity brought about by the multi-machine balancing strategy, variances computed in (27) are smaller than those in (25). The sample data of $P_{\text {loss }}$ and $V_{c}$ are computed from MC simulation and thus the variance and probability can be obtained

$$
\begin{aligned}
& \sigma_{P_{\text {loss }}}^{2}=4.4339 \times 10^{-4} \\
& P\left(P_{\text {loss }}<3.6468\right)=0.9992 \\
& \sigma_{V_{c}}^{2}=3.1261 \times 10^{-7} \\
& P\left(0.98<V_{c}<1.02\right)=0.9914
\end{aligned}
$$

The assessment of variances in (27) has been confirmed by (28). Equation (28) suggests that with the same complementarity condition, the new multi-machine balancing strategy can further improve the probability of qualified transmission loss and system voltage to nearly $100 \%$ by reducing the absolute value of sensitivities for both $P_{\text {loss }}$ and $V_{c}$. This result can be explained by Section II, which derives the relationship between the sensitivity of $P_{\text {loss }}$ and different balancing strategies. It can also be easily comprehended that with more generators to share the power imbalance induced by wind generation, there should be less impacts on system overall steady-state voltage although the number of sharing machines is limited by the labor resource on energy balancing desk in control time-scale.

\section{4) Case D (Application of MTDC with MSC):}

In Case D, MTDC transmission displayed in Fig. 4(b) is employed with MSC at bus DC5 to link the three offshore wind farms. Without loss of generality, Star Topology (ST) based MTDC is selected for the work demonstration, as it has been studies that there is no considerable difference between ST and any other MTDC topologies [16].

The same load condition, balancing strategy and wind speed distribution are applied in Case D. As the MTDC connection is used, the sensitivities of $P_{\text {loss }}$ and $V_{c}$ in respect of three sources of wind power become nearly identical and similar to the sensitivities of the second wind farm in Case C.

$\partial P_{\text {loss }} / \partial P_{w 1}=0.0218, \partial P_{\text {loss }} / \partial P_{w 2}=0.0217, \partial P_{\text {loss }} / \partial P_{w 3}=0.0218$ $\partial V_{c} / \partial P_{w 1}=-0.0009, \partial V_{c} / \partial P_{w 2}=-0.0009, \partial V_{c} / \partial P_{w 3}=-0.0009$

Hence the variance indices are computed to be

$$
\begin{aligned}
& \sigma_{P_{\text {loss }}}^{2}=1.5350 \times 10^{-4} \\
& \sigma_{V_{C}}^{2}=3.0215 \times 10^{-7}
\end{aligned}
$$

Compared with (27), variances in (29) become even smaller as the DC5 with the smallest sensitivity has been selected for MSC. According to the calculated data of $P_{\text {loss }}$ and $V_{c}$ from MC simulation, it can have

$$
\begin{aligned}
& \sigma_{P_{\text {loss }}}^{2}=1.6178 \times 10^{-4} \\
& P\left(P_{\text {loss }}<3.6468\right)=1.0000 \\
& \sigma_{V_{c}}^{2}=3.0620 \times 10^{-7} \\
& P\left(0.98<V_{c}<1.02\right)=0.9916
\end{aligned}
$$

Variances computed in (29) have been validated by (30). Equation (30) indicates that in the scenario of MTDC connection, when wind power changes, the transmission loss will highly unlikely exceed the target loss 3.6468 owing to the further reduction of $\sigma_{P_{\text {loss }}}^{2}$. Meanwhile, the voltage condition becomes more favorable as well when considering the 
uncertainty brought about by wind power. Therefore, it can be concluded from (7) and (9) that MTDC network can certainly alleviate the impact of stochastic fluctuation of wind generation and enhance power system operation significantly by making all the sensitivities of transmission loss and system voltage in respect of wind power identical and small.

5) Case E (DC slack bus at a different location applied to Case D):

Similar to Case D, the ST MTDC and MSC strategy are used. However, DC voltage control has been applied to bus DC6 converter instead. Hence, the sensitivities of $P_{\text {loss }}$ and $V_{c}$ in respect of three wind power sources are similar to the sensitivities of the third wind farm in Case $\mathrm{C}$.

$\partial P_{\text {loss }} / \partial P_{w 1}=0.0539, \partial P_{\text {loss }} / \partial P_{w 2}=0.0538, \partial P_{\text {loss }} / \partial P_{w 3}=0.0539$ $\partial V_{c} / \partial P_{w 1}=-0.0011, \partial V_{c} / \partial P_{w 2}=-0.0011, \partial V_{c} / \partial P_{w 3}=-0.0011$

Hence the variance indices are computed to be

$$
\begin{aligned}
& \sigma_{P_{\text {loss }}}^{2}=9.4000 \times 10^{-4} \\
& \sigma_{V_{c}}^{2}=3.7077 \times 10^{-7}
\end{aligned}
$$

According to the sample data of $P_{\text {loss }}$ and $V_{c}$ obtained from MC simulation, it can have

$$
\begin{aligned}
& \sigma_{P_{\text {loss }}}^{2}=1.0469 \times 10^{-3} \\
& P\left(P_{\text {loss }}<3.6468\right)=0.9798 \\
& \sigma_{V_{c}}^{2}=3.6380 \times 10^{-7} \\
& P\left(0.98<V_{c}<1.02\right)=0.9858
\end{aligned}
$$

The assessment of $\sigma_{P_{\text {loss }}}^{2}$ and $\sigma_{V_{c}}^{2}$ is validated by (32).

Compared with Case $\mathrm{C}$ and $\mathrm{D}$, even with high complementarity level and same balancing strategy, however, the probability of economic and reliable operation drops quite amount of percentages. Hence, it is demonstrated by (32) that MTDC network does not always have a positive contribution to power system operation. In order to improve the economical efficiency and reliability, the converter linked to AC bus with smaller sensitivity of $P_{\text {loss }}$ and $V_{c}$ in respect of offshore wind power (at least smaller than the average sensitivity) should select DC voltage control function, so as to mitigate the influence of the stochastic uncertainty of wind power on the system operation.

\section{6) Case F (Application of MTDC with two types of VDC).}

To replace the MSC, two typical VDC schemes (i.e., PVDC and CVDC) are assessed in Case F and G. Due to the limitation of space, computation results are directly provided for CVDC and Appendix B presents the droop constants in details. The preconditions of Case $\mathrm{F}$ are identical to Case $\mathrm{D}$ and $\mathrm{E}$. When PVDC is employed, the sensitivity of $P_{\text {loss }}$ and $V_{c}$ in respect of three sources of wind power becomes

$$
\partial P_{\text {loss }} / \partial P_{w 1}=0.0375, \partial P_{\text {loss }} / \partial P_{w 2}=0.0374, \partial P_{\text {loss }} / \partial P_{w 3}=0.0375
$$

$\partial V_{c} / \partial P_{w 1}=-0.0010, \partial V_{c} / \partial P_{w 2}=-0.0010, \partial V_{c} / \partial P_{w 3}=-0.0010$

It is understandable that the sensitivities above are still quite similar owing to the similar electrical distance of wind power sources. The sensitivities are between $0.0217(-0.0009)$ and $0.0533(-0.0011)$ since the variation of wind power is shared by each grid-side converter rather than one converter.

Hence the variance indices are computed to be

$$
\begin{aligned}
& \sigma_{P_{\text {loss }}}^{2}=4.5581 \times 10^{-4} \\
& \sigma_{V_{C}}^{2}=3.1111 \times 10^{-7}
\end{aligned}
$$

Variances in (33) are close to that in (27) of Case C since the same complementarity condition is considered and sensitivities here are average, which combine the sensitivity relevant to each grid-side converter together. The sample data of $P_{\text {loss }}$ and $V_{c}$ are computed from $\mathrm{MC}$ simulation, and hence it can have

$$
\begin{aligned}
& \sigma_{P_{\text {loss }}}^{2}=4.5233 \times 10^{-4} \\
& P\left(P_{\text {loss }}<3.6468\right)=0.9992 \\
& \sigma_{V_{c}}^{2}=3.1995 \times 10^{-7} \\
& P\left(0.98<V_{c}<1.02\right)=0.9918
\end{aligned}
$$

The results shown in (33) and (34) are close to each other. By comparing with the variance indices and probabilities calculated in Case $\mathrm{C}$, it can be seen that the impact of MTDC topology on the system operation is not predominant but actually quite limited when using VDC scheme because of the average sensitivity.

The sensitivity and variance indices are also calculated for CVDC scheme and confirmed by MC simulation as follows.

$\partial P_{\text {loss }} / \partial P_{w 1}=0.0376, \partial P_{\text {loss }} / \partial P_{w 2}=0.0374, \partial P_{\text {loss }} / \partial P_{w 3}=0.0376$

$\partial V_{c} / \partial P_{w 1}=-0.0010, \partial V_{c} / \partial P_{w 2}=-0.0010, \partial V_{c} / \partial P_{w 3}=-0.0010$

$\sigma_{P_{\text {loss }}}^{2}=4.5636 \times 10^{-4}, \sigma_{V_{c}}^{2}=3.1112 \times 10^{-7}$

$\sigma_{P_{\text {loss }}}^{2}=4.5280 \times 10^{-4}, \sigma_{V_{c}}^{2}=3.1996 \times 10^{-7}$ (Monte Carlo)

$P\left(P_{\text {loss }}<3.6468\right)=0.9992, P\left(0.98<V_{c}<1.02\right)=0.9916$

Similar calculation results have been noted for both droop control schemes.

\section{7) Case $G$ (A different DC voltage droop constant applied to Case $F)$ :}

Case $\mathrm{G}$ selects smaller droop constant stated in Appendix B. Therefore, sensitivity of $P_{\text {loss }}$ and $V_{c}$ in respect of three wind power sources is computed to be

$\partial P_{\text {loss }} / \partial P_{w 1}=0.0392, \partial P_{\text {loss }} / \partial P_{w 2}=0.0391, \partial P_{\text {loss }} / \partial P_{w 3}=0.0392$

$\partial V_{c} / \partial P_{w 1}=-0.0010, \partial V_{c} / \partial P_{w 2}=-0.0010, \partial V_{c} / \partial P_{w 3}=-0.0010$

The sensitivity has a slight increase as the DC power variation has become increasingly sensitive to the DC voltage change caused by change in wind power.

Hence the indices of variances are computed to be

$$
\begin{aligned}
& \sigma_{P_{\text {loss }}}^{2}=4.9772 \times 10^{-4} \\
& \sigma_{V_{c}}^{2}=3.1202 \times 10^{-7}
\end{aligned}
$$

According to the calculated data of $P_{\text {loss }}$ and $V_{c}$ from $\mathrm{MC}$ simulation, it can be obtained that

$$
\begin{aligned}
& \sigma_{P_{\text {loss }}}^{2}=5.0658 \times 10^{-4} \\
& P\left(P_{\text {loss }}<3.6468\right)=0.9988 \\
& \sigma_{V_{c}}^{2}=3.3047 \times 10^{-7} \\
& P\left(0.98<V_{c}<1.02\right)=0.9914
\end{aligned}
$$

Variance indices are proved by the calculation in (36). Compared with the results of Case F, it is observed that the reduction of DC voltage droop constant has slightly weakened system economic and reliable operation owing to the rise of sensitivity. In other words, power system operation could benefit from the application of MTDC transmission if increasing the DC voltage droop constants.

The results of the sensitivity and variance for CVDC scheme 
are given as follows.

$\partial P_{\text {loss }} / \partial P_{w 1}=0.0430, \partial P_{\text {loss }} / \partial P_{w 2}=0.0429, \partial P_{\text {loss }} / \partial P_{w 3}=0.0430$

$\partial V_{c} / \partial P_{w 1}=-0.0010, \partial V_{c} / \partial P_{w 2}=-0.0010, \partial V_{c} / \partial P_{w 3}=-0.0010$

$\sigma_{P_{\text {loss }}}^{2}=5.9808 \times 10^{-4}, \sigma_{V_{c}}^{2}=3.1404 \times 10^{-7}$

$\sigma_{P_{\text {loss }}}^{2}=6.0375 \times 10^{-4}, \sigma_{V_{c}}^{2}=3.3169 \times 10^{-7}$ (Monte Carlo)

$P\left(P_{\text {loss }}<3.6468\right)=0.9966, P\left(0.98<V_{c}<1.02\right)=0.9912$

From the sensitivity calculation above, it can be observed that when droop constant is small, the variation of wind power generation has more significant effects on the transmission loss in the case of CVDC due to the characteristic of bipole DC grid.

\section{Discussions}

The PDF variance could be proposed as an index as it is the key factor to affect the shape of PDF and hence the probability of the economic and reliable margins. Given the same preconditions, as proved in the case study, the smaller/bigger variance will lead to bigger/smaller probabilistic operational margin. Therefore, by using the cumulant-based theory to estimate the variances, an explicit relationship between the two operational margins (i.e., economical efficiency and reliability) and four impact factors of offshore wind farms (i.e., complementarity, balancing, topology and control scheme) can be revealed. The accuracy of analytical estimation of the variances (that could accommodate the cases with or without correlations) is also verified by MC simulation in the case study

To sum up, the proposed variance indices generally have two major practical applications: 1. to predict the probabilistic operational margin and assess the feasibility and quality of the integration plans of offshore wind farms in a preliminary manner without running time-consuming $\mathrm{MC}$ simulation (although the indices cannot give a very accurate probability of economic and reliable operational margin, at least they can be employed to rank different integration plans based on the impact of plans on the economical efficiency and reliability of system operation); 2. to provide an explicit expression of PDF variance of two probabilistic operational margins with respect to the four key integration aspects of offshore wind power generation and reveal their inner connections. Once the accurate probabilistic operational margins are obtained, the closed-form solution of variance indices can facilitate the system planner's understanding on why and how different conditions of the four key integration aspects of offshore wind farms contribute to a higher or lower economical efficiency and reliability and thus impact the system operation, so that some possible adjustments can be done to the integration plans in order to reduce more operational cost and maintain better supply quality. In particular, the value of the second application should be emphasized because it is not a numerical analysis by trials but an analytical approach to explain the mechanism of the inner connection.

\section{CONCLUSIONS}

Power system economic and reliable operation is intensively studied in the paper considering the effect of complementarity level, power balancing strategy, HVDC transmission topology and grid control scheme of the offshore wind farms. The PDF variance index of transmission loss and voltage of critical node assessed by cumulant-based method with a closed-form solution is derived for the first time as an efficient tool in power system planning and operation as discussed in the previous section. Some key conclusions from the seven sequential study cases are drawn as follows:

1. With low level of complementarity from composite wind active power, power system has a high probability to breach the standard limits of economic and reliable operation and the uncertainty of wind power could weaken system voltage even at the remote end.

2. Complementarity of offshore wind generation can be utilized to enhance the economical efficiency and reliability regardless of the HVDC transmission topologies.

3. It is generally beneficial to have more conventional machines to share the power imbalance caused by the variable wind power if achievable in real time, as this balancing strategy can increase the probability of system economic and reliable operation.

4. The DC voltage control function should locate close to the AC bus with smaller sensitivity of transmission loss and voltage magnitude in respect of wind active power to mitigate the influence of stochastic uncertainty brought by wind power on the system operation if MTDC MSC scheme is employed.

5. VDC is more robust in reducing the loss and maintaining system voltage within standard limits compared with MSC. Higher droop constant can enhance the effectiveness in suppressing the stochastic uncertainty.

6. Comparing the two VDC, PVDC shows more robustness to droop constant change due to the applied bipole network.

\section{APPENDIX}

\section{A. Wind Power Generator Model and Wind Distribution Parameters}

A.1 Wind Power Generator

A 70MW DFIG model [23] is used for all three wind farms and the parameters in p.u. are

$X_{s}=X_{r}=0.29, X_{m}=2.6, R_{s}=0.0, R_{r}=0.0013, P_{w 0}=3.3333$

A.2 Wind Speed Distribution

$\mu=6 \mathrm{~m} / \mathrm{s}, \sigma=2.5 \mathrm{~m} / \mathrm{s}($ Case A $), \sigma=2.0 \mathrm{~m} / \mathrm{s}($ Case B-G)

$\rho=\left[\begin{array}{ccc}1 & 0.9 & 0.3 \\ 0.9 & 1 & 0.5 \\ 0.3 & 0.5 & 1\end{array}\right]\left(\right.$ Case A), $\rho=\left[\begin{array}{ccc}1 & 0.5 & 0 \\ 0.5 & 1 & 0 \\ 0 & 0 & 1\end{array}\right]$ (Case B-G)

A.3 Power-Wind Speed Curve Data

$v_{\text {cut-in }}=4 \mathrm{~m} / \mathrm{s}, v_{\text {rated }}=10 \mathrm{~m} / \mathrm{s}, v_{\text {furling }}=22 \mathrm{~m} / \mathrm{s}, P_{\text {rated }}=1 \mathrm{p} . \mathrm{u}$.

\section{B. VSC-HVDC Network Parameters}

VSC-HVDC network data in p.u. are

$R_{14}=R_{25}=R_{36}=0.0006($ Case $A)$

$R_{17}=R_{37}=R_{47}=R_{67}=0.0007, R_{27}=R_{57}=0.0003($ Case $B-G)$ where bus 7 is the central terminal of Star Topology. The 
parameters of VSC model in p.u. are

$R_{c}=0.0001, X_{c}=0.1643, R_{t}=0.0015, X_{t}=0.1121$,

$B_{f}=0.0887$

The constants of DC voltage droop [15] in Case F and G are $k_{\text {droop }}=0.04($ Case F $), 0.004($ Case $\mathrm{G})$

\section{ACKNOWLEDGMENT}

The authors would like to acknowledge the support of Department of Electrical Engineering, Hong Kong Polytechnic University for the Start-up Fund Research Project (1-ZE68).

\section{REFERENCES}

[1] E. Kahn, "The reliability of distributed wind generators," Electric Power Systems Research, vol. 2, pp. 1-14, Mar. 1979.

[2] C. L. Archer and M. Z. Jacobson, "Supplying baseload power and reducing transmission requirements by interconnecting wind farms," Journal of Applied Meteorology and Climatology, vol. 46, pp. 1701-1717, 2007.

[3] W. Kempton, F. M. Pimenta, D. E. Veron, and B. A. Colle, "Electric power from offshore wind via synoptic-scale interconnection,"Proceedings of the National Academy of Sciences, vol. 107, pp. 7240, 2010.

[4] Y. Liu, L. Xiao and H. Wang, "Investigation on the spatiotemporal complementarity of wind energy resources in China," Science China Technological Sciences, vol. 55, no.3, pp. 725-734, 2012.

[5] Y. Liu, L. Xiao and H. Wang, "Analysis on the hourly spatiotemporal complementarities between China's solar and wind energy resources spreading in a wide area," Science China Technological Sciences, vol. 56, no.3, pp. 683-692, 2013

[6] S. Q. Bu, W. Du, and H. F. Wang, "Investigation on Probabilistic SmallSignal Stability of Power Systems as Affected by Offshore Wind Generation," IEEE Trans. on Power Systems, vol. 30, no. 5, pp. 2479 2486, Sept. 2015.

[7] P. Zhang, and S. T. Lee, "Probabilistic Load Flow Computation Using the Method of Combined Cumulants and Gram-Charlier Expansion," IEEE Trans. on Power Systems, vol. 19, no. 1, pp. 676-682, Feb. 2004.

[8] Z. C. Hu, and X. F. Wang, "A Probabilistic Load Flow Method Considering Branch Outages," IEEE Trans. on Power Systems, vol. 21, no. 2, pp. 507-514, May 2006

[9] Y. Y. Hong, and Y. F. Luo, "Optimal VAR Control Considering Wind Farms Using Probabilistic Load-Flow and Gray-Based Genetic Algorithms," IEEE Trans. on Power Systems, vol. 24, no. 3, pp. 14411449, July 2009.

[10] A. Schellenberg, W. Rosehart, and J. Aguado, "Cumulant-Based Probabilistic Optimal Power Flow (P-OPF) With Gaussian and Gamma Distrbutions," IEEE Trans. on Power Systems, vol. 20, no. 2, pp. 773-781, May 2005.

[11] A. Tamtum, A. Schellenberg, and W. Rosehart, "Enhancements to the Cumulant Method for Probabilistic Optimal Power Flow Studies," IEEE Trans. on Power Systems, vol. 24, no. 4, pp. 1739-1746, Nov. 2009.

[12] T. Ackermann, "Transmission systems for offshore wind farms," IEEE Power Engineering Review, vol. 22, no. 12, pp. 23-27, Dec. 2002.

[13] J. Beerten, S. Cole and R. Belmans, "Generalized Steady-state VSC MTDC Model for Sequential AC/DC Power Flow Algorithms," IEEE Trans. on Power Systems, vol. 27, no. 2, pp. 821-829, May 2012.

[14] N. R. Chaudhuri, R. Majumder, B. Chaudhuri, J. Pan and R. Nuqui, "Modeling and Stability Analysis of MTDC Grids for Offshore Wind Farms: A Case Study on the North Sea Benchmark System," IEEE Power and Energy Society General Meeting, pp. 1-7, 2011.

[15] T. M. Haileselassie and K. Uhlen, "Impact of DC Line Voltage Drops on Power Flow of MTDC Using Droop Control," IEEE Trans. on Power Systems, vol. 27, no. 3, pp. 1441-1449, Aug. 2012.

[16] O. Gomis-Bellmunt, J. Liang, J. Ekanayake, R. King and N. Jenkins, "Topologies of multiterminal HVDC-VSC transmission for large offshore wind farms," Electric Power Systems Research, vol. 81, pp. 271-281, Oct. 2010.

[17] J. Cao, W. Du, H. F. Wang and S. Q. Bu, "Minimizatoin of Transmission Loss in Meshed AC/DC Grids With VSC-MTDC Networks," IEEE Trans. on Power Systems, vol. 28, no. 3, pp. 3047-3055, Aug. 2013.
[18] M. Kendall, Kendall's Advanced Theory Statistics. New York (NY USA): Oxford University Press, 1987.

[19] H. Cramer, Numerical Methods of Statistics. Princeton, NJ: Princeton University Press, 1946.

[20] National Grid Electricity Plc Transmission Losses Report, available online.

[21] National Electricity Transmission System Security and Quality of Supply Standards, available online.

[22] G. Rogers, Power System Oscillations. Kluwer Academic Publishers, 2000.

[23] H. Akagi and H. Sato, "Control and Performance of a Doubly-Fed Induction Machine Intended for a Flywheel Energy Storage System," IEEE Trans. on Power Electronics, vol. 17, no. 1, pp. 109-116, Jan. 2002.

[24] G. Papaefthymiou and D. Kurowicka, "Using Copulas for Modeling Stochastic Dependence in Power System Uncertainty Analysis," IEEE Trans. on Power Systems, vol. 24, no. 1, pp. 40-49, Feb. 2009.

\section{BIOGRAPHIES}

S. Q. Bu received the Ph.D. degree from the electric power and energy research cluster, the Queen's University of Belfast, Belfast, U.K., in 2012, where he continued his postdoctoral research work in the same department before entering industry. Subsequently, he joined National Grid UK as a Power System Engineer and then became an experienced UK National Transmission System Planner and Operator, during which time he has been awarded various prizes due to excellent performances and outstanding contributions in operational and commissioning projects. He is an Assistant Professor with Hong Kong Polytechnic University and also a Chartered Engineer with UK Engineering Council.

His research interests are power system stability analysis and operation control, including wind power generation, PEV, HVDC, FACTS and ESS.

W. Du (M'14) received the PhD degree from the University of Bath, Bath, U.K., in 2009. She is now a full professor with the School of Electrical and Electronic Engineering, North China Electric Power University, Beijing, China. Her main research interests are power system stability analysis and control, including energy storage systems, FACTS, EV, and renewable power generations.

H. F. Wang (M'96-SM'02) is a full professor with the School of Electrical and Electronic Engineering, Northern China Electric Power University, Beijing, China. He had worked in the UK for years and was the Chair in Electrical Engineering and full professor at the Queen's University of Belfast, UK, before he moved to Beijing. His main research interests are power system stability analysis and control, including energy storage systems, FACTS, EV, and renewable power generations.

Y. Liu received the Ph.D. degree from Institute of Electrical Engineering, Chinese Academy of Sciences, China, in 2015. He is currently working with the Institute of Electrical Engineering, Chinese Academy of Sciences, China. His research interests revolve around power system economics and stability, including wind energy, energy storage, and HVDC technology.

X. Liu (M'12) received the Ph.D. degree in electrical and electronic engineering from Queen's University Belfast (QUB), Belfast, U.K., in 2009 Her Ph.D. work on algorithm development in 'moving window kernel PCA for adaptive monitoring of nonlinear processes' is ranked third among the most cited CILS journal articles published since 2009. Currently, she is a Lecturer with the Energy, Power and Intelligent Control Cluster at QUB. Her research interests include the development of advanced data-analysis tools for smart-grid wide-area monitoring and protective control. 